\title{
Robust discovery of partial differential equations in complex situations
}

\author{
$\mathrm{Hao} \mathrm{Xu}^{1, *}$ and Dongxiao Zhang $\mathbb{1}^{2,3, \dagger}$ \\ ${ }^{1}$ Beijing Innovation Center for Engineering Science and Advanced Technology (BIC-ESAT), Energy \& Resources Engineering (ERE), \\ and State Key Laboratory for Turbulence and Complex Systems (SKLTCS), College of Engineering, \\ Peking University, Beijing 100871, People's Republic of China \\ ${ }^{2}$ School of Environmental Science and Engineering, Southern University of Science and Technology, \\ Shenzhen 518055, People's Republic of China \\ ${ }^{3}$ Intelligent Energy Lab, Peng Cheng Laboratory, Shenzhen 518000, People's Republic of China
}

(Received 30 May 2021; accepted 30 August 2021; published 21 September 2021)

\begin{abstract}
Data-driven discovery of partial differential equations (PDEs) has achieved considerable development in recent years. Several aspects of problems have been resolved by sparse regression-based and neural-network-based methods. However, the performance of existing methods lacks stability when dealing with complex situations, including sparse data with high noise, high-order derivatives, and shock waves, which bring obstacles to calculating derivatives accurately. Therefore, a robust PDE discovery framework, called the robust deep-learning genetic algorithm (R-DLGA), that incorporates the physics-informed neural network (PINN) is proposed in this paper. In the framework, preliminary results of potential terms provided by the DLGA are added into the loss function of the PINN as physical constraints to improve the accuracy of derivative calculation. It assists in optimizing the preliminary result and obtaining the ultimately discovered PDE by eliminating the error compensation terms. The stability and accuracy of the proposed R-DLGA in several complex situations are examined for proof and concept, and the results prove that the proposed framework can calculate derivatives accurately with the optimization of the PINN and possesses surprising robustness for complex situations, including sparse data with high noise, high-order derivatives, and shock waves.
\end{abstract}

DOI: 10.1103/PhysRevResearch.3.033270

\section{INTRODUCTION}

Recently, data-driven discovery of partial differential equations (PDEs) has been studied to explore additional possibilities for discovering underlying governing equations from available data. Sparse regression-based techniques, including least absolute shrinkage and selection operator (LASSO) [1], sequential threshold ridge regression (STRidge) [2], sparse Bayesian [3], sparse identification of nonlinear dynamics (SINDy) [4], and other methods evolving from them [5-8], provide a paradigm for PDE discovery by identifying sparse PDE terms from a predetermined candidate library which contains several potential terms. Meanwhile, deep-learning techniques, including the physics-informed neural network (PINN) [9,10], the PDE network (PDE-NET) [11], and the deep-learning PDE (DL-PDE) [12], are employed to improve the accuracy of derivative calculation via automatic differentiation of the neural network [13], which is crucial to the success of PDE discovery with noisy or sparse data. Unfor-

\footnotetext{
*390260267@pku.edu.cn

†zhangdx@ sustech.edu.cn
}

Published by the American Physical Society under the terms of the Creative Commons Attribution 4.0 International license. Further distribution of this work must maintain attribution to the author(s) and the published article's title, journal citation, and DOI. tunately, although the abovementioned methods work well with a small but complete candidate library, they are not sufficiently flexible to handle PDEs with unusual terms that require the candidate library to contain numerous terms to remain complete. For example, the Chaffee-Infante equation with the unusual term $u^{3}$ has proven to be a challenge for sparse-regression methods since the candidate library that contains the unusual term is large, and it is hard to maintain parsimony of the result [14]. Therefore, genetic algorithm (GA)-based methods become a superior choice for PDE discovery since they can generate a large variation library via crossover and mutation from a few basic genes [14-17]. Based on these fundamental techniques, more difficult problems are further solved to approach practical applications more closely. For example, parametric PDEs were successfully discovered by $\mathrm{Xu}$ et al. [18] and Rudy et al. [19] through a stepwise deep-learning GA (stepwise DLGA) and sequential grouped threshold ridge regression (SGTR), respectively. Discovery of stochastic PDEs was accomplished by Bakarji and Tartakovsky [20] through transforming PDEs to probability density functions. However, the performances of extant methods lack stability when dealing with complex situations, including sparse data with high noise, high-order derivatives, and PDEs with shock waves.

Practically, experimental data are often precious and scarce and may contain a certain level of noise due to inevitable systematic errors in the experiment, which may pose a challenge to PDE discovery. Although some of the abovementioned 
methods have achieved satisfactory performances when faced with a high level of noise, such as $25 \%$ noise [13,14] or even $50 \%$ in some cases [21], many data points are required to cope with high noise. Meanwhile, discovery of PDEs from sparse data also necessitates relatively clean data with low noise. In other words, it is difficult to handle sparse data with high noise. Another problem is the identification of PDEs with high-order derivatives, such as the Korteweg-de Vries (KdV) equation and the Kuramoto-Sivashinsky (KS) equation, since the accuracy of calculation of high-order derivatives decreases rapidly with the increase of noise level. Furthermore, a large amount of data is necessary to maintain the precision of derivative calculation. To handle this problem, authors of recent works have attempted to discover the integral formation of PDEs as an alternative approach, which can decrease the required derivative order [22,23]. However, it is challenging to deal with PDEs that cannot be converted to integral form, and the integration process is time intensive. Messenger and Bortz [24] have proposed a framework, called weak SINDy, which employs the weak form to eliminate pointwise derivative approximations and obtain satisfactory outcomes on several PDEs and ordinary differential equations with high levels of noise. However, a large amount of data is required, and the algorithm is less robust for data with limited cycles. The final challenge is PDEs with shock waves, which exist in many physical processes, especially in the field of gas and fluid dynamics. At the position of the shock wave, the physical quantities change dramatically, which leads to a great deviation in derivative calculations and finally incurs failure in PDE discovery.

All the challenges mentioned above are concentrated on one core issue: derivative calculation. Consequently, determination of how to maintain the accuracy of derivative calculation in complex conditions has become a vital problem because merely relying on finite difference or automatic differentiation is not sufficiently accurate to handle diversified situations. Regarding this issue, Both et al. [25] point out a promising approach to increase the accuracy of derivative calculation by employing the PINN to further optimize gradients. In their work, the outcome of LASSO is added into the loss function of the PINN as physical constraints to improve the process of optimization, and results demonstrate that the derivative calculation is more accurate and more robust to sparse and noisy data. However, this method is unstable when facing high noise because the outcome is sensitive to the selection of the threshold which is employed to obtain sparsity. In addition, the candidate library in this work only contains 12 terms because it is difficult to maintain sparsity of the outcome when the size of the candidate library is large. In other words, although the proposed method can improve the accuracy of derivative calculation, it is not sufficiently stable for practical utilization. Therefore, it is essential to search for a robust method to discover PDEs in complex situations.

To deal with the problem of robustness and accuracy in complex situations, a method called the robust DLGA (RDLGA) is proposed, which employs the DLGA to obtain a preliminary result of potential terms from a large variation library. It then utilizes these potential terms as physical constraints of the PINN to further modify the derivatives, which assists in optimizing the identified potential terms by eliminat- ing the error compensation terms. Our proposed method does not require a predetermined complete candidate library with numerous terms because additional potential terms can be augmented by the DLGA. Consequently, it is easier to obtain sparsity in the process of the PINN and thereby increase the stability of the method. The proposed method is tested for proof of concept with several cases in complex situations that are difficult to be resolved by existing methods, including the $\mathrm{KdV}$ equation with sparse and noisy data, the KS equation with high-order derivatives, and the Burgers equation with shock waves. Satisfactory outcomes are obtained, and our proposed method maintains accuracy and robustness in these complex situations.

The remainder of this paper proceeds as follows. Section II establishes the framework of the R-DLGA. Section III presents the performance of PDE discovery with our proposed algorithm in complex conditions and illustrates the robustness and accuracy of the R-DLGA. Finally, Sec. IV summarizes the advantages and disadvantages of the proposed algorithm and provides remarks and recommendations for future work.

\section{METHODS}

\section{A. Problem settings}

For PDE discovery, our goal is to discover the form of underlying governing equations from spatial-temporal observation data, which is given as $u(x, t)$. The form of the PDE can be written as follows:

$$
u_{t}=F\left(u, u_{x}, u u_{x}, u^{2} u_{x}, u_{x x}, \ldots ; \vec{\xi}\right),
$$

where $F$ is an operator that indicates a linear combination of terms, and $\vec{\xi}$ is the coefficient vector. With available observation data $u\left(x_{i}, t_{i}\right)$, this issue can be converted to a regression problem that is described as

$$
\left[\begin{array}{c}
u_{t}\left(x_{1}, t_{1}\right) \\
u_{t}\left(x_{2}, t_{2}\right) \\
\vdots \\
u_{t}\left(x_{N}, t_{N}\right)
\end{array}\right]=\left[\begin{array}{cccc}
u\left(x_{1}, t_{1}\right) & \cdots & u u_{x}\left(x_{1}, t_{1}\right) & \cdots \\
u\left(x_{2}, t_{2}\right) & \cdots & u u_{x}\left(x_{2}, t_{2}\right) & \cdots \\
\vdots & \ddots & \vdots & \ddots \\
u\left(x_{N}, t_{N}\right) & \cdots & u u_{x}\left(x_{N}, t_{N}\right) & \cdots
\end{array}\right] \cdot \vec{\xi},
$$

where $N$ is the data volume, and $\vec{\xi}$ is the coefficient vector. Equation (2) can be abbreviated as

$$
U_{t}=\Phi \cdot \vec{\xi},
$$

where $U_{t}$ refers to the left-hand side term, and $\Phi$ refers to the matrix of the potential terms. It is worth noting that $\Phi$ may contain a large variety of potential terms, and thus, the coefficient vector $\xi$ is a sparse vector with most of the elements being zero. For PDE discovery, we aim to identify nonzero terms and corresponding coefficients from data. Therefore, the difficulty of the problem lies in how to consider both accuracy and parsimony of the discovered PDE.

\section{B. DLGA}

In our earlier work, an algorithm combining deep learning and a GA, called the DLGA, was proposed to discover PDEs [14]. Although it manages to discover many common PDEs, it is unstable in complex situations, as mentioned in Sec. I. 

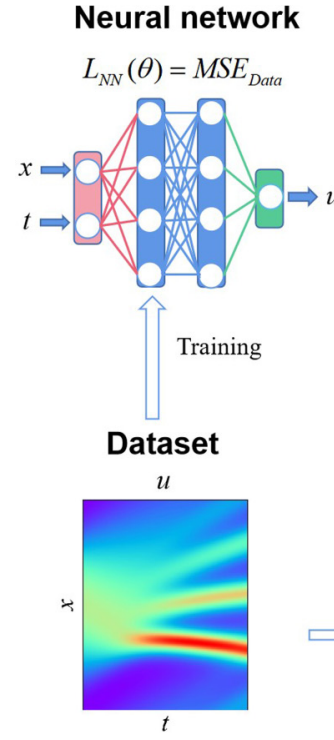

Generalized genetic algorithm

Discovered potential PDE terms

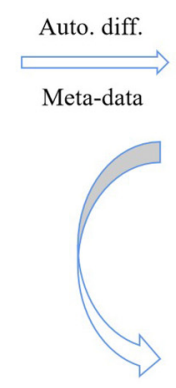

$\{[(0,1): 0],[(3): 0],[(2): 3]\}$

$\mathbb{1}$

$u_{t}, u u_{x}, u_{x x x}, u_{x x x x x}$

PDE terms

Physics-informed (a)

(b)

neural network

$L_{P N N}(\theta)=\lambda_{\text {Data }} M S E_{\text {Data }}+\lambda_{P D E} M S E_{P D E}$

(a)

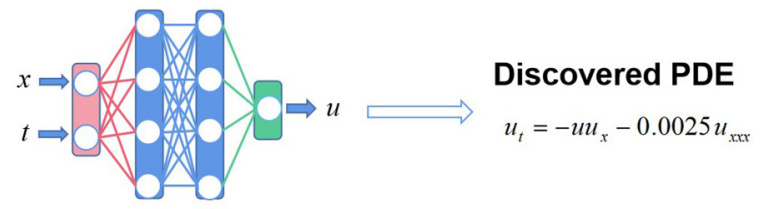

FIG. 1. Workflow of the robust deep-learning genetic algorithm (R-DLGA), including (a) the DLGA steps and (b) the physics-informed neural network (PINN) steps. In the DLGA steps, the neural network is utilized to construct a surrogate model from available data, and then the generalized genetic algorithm is employed to identify potential terms. In the PINN steps, the discovered potential terms are added into the loss function $L_{\mathrm{PINN}}(\theta)$ as physical constraints to further optimize the derivatives and discover the ultimate partial differential equation (PDE).

In this paper, a generalized GA is proposed based on the DLGA framework to give a preliminary result of potential terms that is relatively parsimonious and contains certain physical information. The workflow of our proposed R-DLGA is demonstrated in Fig. 1. The DLGA steps are illustrated in Fig. 1(a), and the PINN steps are illustrated in Fig. 1(b). In the DLGA process, a neural network is employed to construct a substitute model from available observation data. Then a large amount of metadata is generated on a regular spatial-temporal grid, and automatic differentiation is utilized to calculate the derivatives of the metadata. Finally, the generalized GA is utilized to give a preliminary result of potential terms. In this part, the procedure of DLGA steps, including the neural network and generalized GA, is introduced in detail.

\section{Neural network}

In this paper, the neural network is a fully connected artificial neural network, which is composed of an input layer, an output layer, and several hidden layers. The input of the neural network is the spatial-temporal location $(x, t)$, and the output is $\mathrm{NN}(x, t ; \theta)$. Here, $\theta$ is the parameter of the neural network, including weights and bias. The neural network is trained by minimizing the loss function $L_{\mathrm{NN}}(\theta)$, which is written as

$$
L_{\mathrm{NN}}(\theta)=\mathrm{MSE}_{\text {Data }}=\frac{1}{N} \sum_{i=1}^{N}\left[u\left(x_{i}, t_{i}\right)-\mathrm{NN}\left(x_{i}, t_{i} ; \theta\right)\right]^{2},
$$

where $N$ is the data volume. An early termination technique is employed to prevent overfitting. The trained neural network functions as a surrogate model for the underlying physical system and is utilized to generate metadata on a spatial-temporal grid. Meanwhile, automatic differentiation of the neural network is employed to calculate derivatives of the metadata, which has been proven to be more robust to data noise.

\section{Generalized GA}

The GA is an optimization algorithm that simulates the evolutionary process of natural selection, which is usually comprised of digitization, crossover, mutation, fitness calculation, and evolution. In this paper, a GA called the generalized $\mathrm{GA}$ is proposed. Different from the common GA, the generalized GA attempts to consider PDE terms in compound form that consists of an inner term and a derivative order. In this way, PDE terms can be expressed flexibly in a general form since high-order derivatives (e.g., fifth-order derivative) and complex terms [e.g., $\left(u^{2} u_{x}\right)_{x x}$ ] can be reached by crossover and mutation without prior definition. Meanwhile, benefited from the compound form, it is easier for the generalized GA to obtain parsimony since a compound form can represent a combination of several single forms. In this part, the procedure of our proposed generalized GA for PDE discovery is introduced in detail.

(a) Digitization. In this paper, a unique digitization technique is advanced to express possible terms clearly and flexibly in general form. The principle of digitization is illustrated in Fig. 2. Different from previous works $[14,17]$ that only consider the simple form of terms (e.g., $u u_{x}$ ), our proposed digitization technique manages to express terms in compound form [e.g., $\left(u^{2}\right)_{x x}$ ]. Considering the existence of the weak form of PDEs, most PDE terms can be written in compound form, which is composed of an inner term and a derivative order. The relation between PDE terms in compound form and genetic form is illustrated in Fig. 2(a). It is worth mentioning that our proposed digitization method is directly abstract from the composition of compound terms, which is more intuitive and easier to understand.

The gene is the basic unit of the GA, in which numbers are employed to represent the derivatives of corresponding order. Here, basic genes are defined up to third-order derivatives to form the inner term, which is represented by parentheses. It is 
(a)

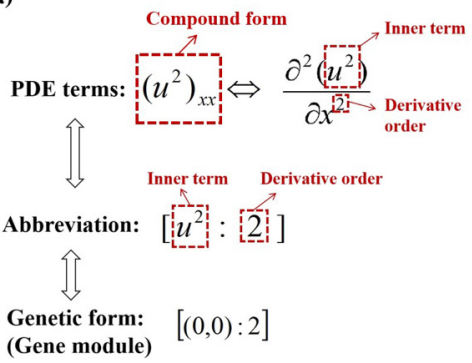

(b)

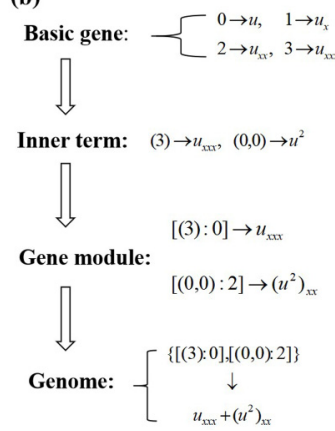

FIG. 2. The principle of digitization in the generalized genetic algorithm. (a) The relation between partial differential equation (PDE) terms in compound form and genetic form; (b) the digitization process from basic gene to genome.

assumed that there is only multiplication of basic genes in the inner term. For example, $(0,2)$ refers to the inner term $u u_{x x}$. With the inner term and the derivative order, the compound form of PDE terms can be established in square brackets, which is called the gene module. In the gene module, the inner term and the derivative order are separated by colons. Since each gene module represents a corresponding PDE term, the PDE can be expressed as the addition of multiple terms, which is called the genome. The genome is represented by curly brackets, and different gene modules are separated by commas. The whole digitization process from basic gene to genome is displayed in Fig. 2(b). It is worth noting that the metadata generated by the trained neural network $\mathrm{NN}(x, t ; \theta)$ are utilized here, derivatives in basic genes are calculated by automatic differentiation, and the value of the inner term can be calculated easily by multiplication. Meanwhile, the value of the PDE term in compound form is calculated by the finite difference method since the value of the inner term is calculated on a regular grid. In this manner, the calculation of PDE terms in compound form, which is unsettled in previous works $[14,17]$, is resolved in this paper. With this unique digitization technique, the compound form of terms can be expressed, which will assist in obtaining a compact and parsimonious result in the generalized GA.

(b) Crossover and mutation. Crossover and mutation are crucial to the GA because they can generate new genomes and thereby expand the search scope, which leads to a large variation library of terms. A diagrammatic sketch of crossover and mutation is provided in Fig. 3. In the crossover process, two parent genomes exchange certain gene modules to produce their children, which enables the gene module in the parent genomes to transfer to the next generation. In the mutation process, the genome randomly mutates to a new genome. In this paper, there are four ways of mutation, including deletemodule mutation, basic gene mutation, order mutation, and add-module mutation. For delete-module mutation, a gene module is randomly deleted. For basic gene mutation, one random gene in the gene module is mutated by decreasing 1 from the gene number. Particularly, 0 is mutated to be 3 . For example, $(1,0)$ may be mutated into $(0,0)$, and $(0,2)$ may be mutated into $(3,2)$. For order mutation, the derivative order in a certain gene module is mutated in the same way as basic gene

(a)

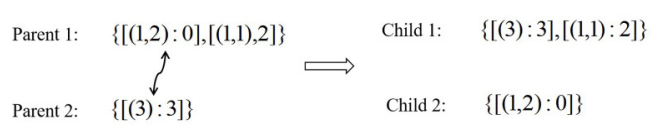

(b)

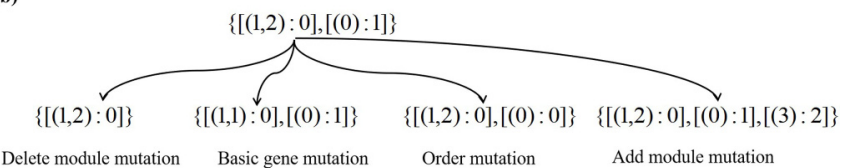

FIG. 3. Diagrammatic sketch of (a) crossover and (b) mutation in the generalized genetic algorithm.

mutation. For add-module mutation, a random gene module is added into the genome.

(c) Fitness calculation. In the GA, the quality of the genome is determined by the fitness function, which is defined as follows:

$$
F=\mathrm{MSE}+\varepsilon \sum_{k=1}^{N_{\mathrm{GM}}} L\left(\mathrm{GM}_{k}\right)
$$

with

$$
\operatorname{MSE}=\frac{1}{N_{x} N_{t}} \sum_{j=1}^{N_{t}} \sum_{i=1}^{N_{x}}\left|U_{t}\left(x_{i}, t_{j}\right)-\vec{\beta} U_{\mathrm{R}}\left(x_{i}, t_{j}\right)\right|^{2},
$$

where $F$ denotes the fitness. The fitness contains two parts, including the mean squared error (MSE) part and the penalty part. Here, MSE is calculated according to Eq. (6), where $U_{t}\left(x_{i}, t_{j}\right)$ is the value of the left-hand term with the size of $1 \times 1 ; U_{\mathrm{R}}\left(x_{i}, t_{j}\right)$ is the value of right-hand terms translated from the genome, which is a $n \times 1$ vector; $n$ is the number of PDE terms; $N_{x}$ and $N_{t}$ are the number of $x$ and $t$ of the metadata, respectively; and $\vec{\beta}$ with the size of $1 \times n$ is the coefficient for the right-hand terms which is calculated by least square regression. In the penalty part, $\varepsilon$ is a hyperparameter to control the weight of the penalty. In this paper, $\varepsilon$ is determined by experience, but it is worth noting that the selection of $\varepsilon$ is not strict since the discovered potential terms can be further optimized in the PINN step. Here, $N_{\mathrm{GM}}$ denotes the number of gene modules in the genome, and $L\left(\mathrm{GM}_{k}\right)$ refers to the length of the $k$ th gene module in the genome, i.e., the number of genes in the inner term. For example, the length of gene module [(1,2):0] is 2 , and the length of gene module $[(1,2,3): 2]$ is 3 .

The MSE part in the fitness function is utilized to reflect the accuracy of the discovered PDE. Specifically, the smaller the MSE, the more accurate the discovered PDE. However, considering that a parsimonious form is expected to be discovered, a penalty is needed to be added into the fitness to avoid overfitting. The penalty part in Eq. (5) limits the total length of the genome, which enables the discovered PDE to tend to be parsimonious. Therefore, in this paper, the smaller the fitness function, the better the genome.

(d) Evolution. The workflow for the whole evolution process in the generalized GA is presented in Fig. 4. In this paper, genomes are randomly generated based on basic genes as the initial parent generation. Then each parent genome crosses 


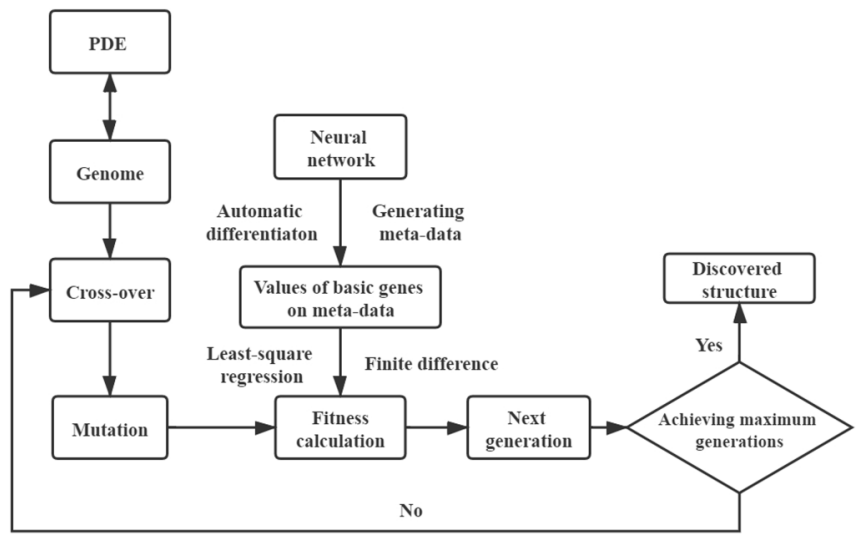

FIG. 4. The workflow for the whole evolution process in the generalized genetic algorithm.

over twice to produce twice the number of children (e.g., 200 parents produce 400 children). Afterward, four types of mutation occur independently, and the fitness of the children is calculated. The best half of the children with less fitness in each generation are reserved to be the subsequent parent generation, while the others are dropped. This cycle continues until achieving maximum generations, and the best children in the final generation are the ultimate identified potential terms. A strategy is employed here to accelerate the convergence, in which the best child in each generation is fixed until a better one occurs to replace it.

\section{PINN}

After the DLGA process, a preliminary result of potential terms is obtained, which may be imprecise in complex conditions but still contains some physical information. Therefore, the identified potential terms can be added into the loss function of the neural network $\mathrm{NN}(x, t ; \theta)$ to provide physical constraints and construct a $\operatorname{PINN}(x, t ; \theta)[9,10]$. The structure, the weights, and the bias of the $\operatorname{PINN}(x, t ; \theta)$ are the same as those of the trained neural network $\mathrm{NN}(x, t ; \theta)$, which means that $\operatorname{PINN}(x, t ; \theta)$ is trained on the basis of $\mathrm{NN}(x, t ; \theta)$. The input is also the same spatial-temporal locations $(x, t)$, and the output is the $\operatorname{PINN}(x, t ; \theta)$. The loss function of $\operatorname{PINN}(x, t ; \theta)$ is

$$
\begin{aligned}
L_{\mathrm{PINN}}(\theta)= & \lambda_{\text {Data }} \mathrm{MSE}_{\text {Data }}+\lambda_{\mathrm{PDE}} \mathrm{MSE}_{\mathrm{PDE}} \\
= & \frac{\lambda_{\text {Data }}}{N} \sum_{i=1}^{N}\left[u\left(x_{i}, t_{i}\right)-\operatorname{PINN}\left(x_{i}, t_{i}\right)\right]^{2} \\
& +\frac{\lambda_{\mathrm{PDE}}}{N} \sum_{i=1}^{N}\left|U_{t}\left(x_{i}, t_{i}\right)-\vec{\beta}_{P} U_{P}\left(x_{i}, t_{i}\right)\right|^{2},
\end{aligned}
$$

where $N$ is the number of observation data, $\mathrm{MSE}_{\mathrm{data}}$ is the same as that in Eq. (4), and MSE $E_{\mathrm{PDE}}$ is the MSE between the left-hand side term and the potential terms on the right-hand side. In this paper, $\lambda_{\text {Data }}$ and $\lambda_{\mathrm{PDE}}$, which are corresponding coefficients of the two constraint terms, are both set to be 1. It is worth noting that these coefficients can be adjusted according to the magnitude of $\mathrm{MSE}_{\text {Data }}$ and $\mathrm{MSE}_{\mathrm{PDE}}$ for better outcomes. Furthermore, other constraint terms, such as boundary or initial conditions and engineering controls, may be included in Eq. (7) [26] if relevant prior information is known, and the weighting coefficients for these constraints may be determined automatically [27]. In Eq. (7), $U_{t}\left(x_{i}, t_{i}\right)$ is the value of the left-hand side term, and $U_{P}\left(x_{i}, t_{i}\right)$ is the value of preliminary potential terms identified by the DLGA with the size of $n_{p} \times 1$, where $n_{p}$ is the number of potential terms, which is like that in Eq. (2), and $\vec{\beta}_{P}$ with the size of $1 \times n_{p}$ is the coefficient for the right-hand terms. In each training epoch, $U_{t}\left(x_{i}, t_{i}\right)$ and $U_{P}\left(x_{i}, t_{i}\right)$ can be calculated easily via automatic differentiation. Therefore, the coefficient vector $\vec{\beta}_{P}$ can be obtained by regression techniques. For the first 1000 epochs, the LASSO regression technique [1] is employed, which is written as

$$
\vec{\beta}_{P}=\operatorname{argmin}_{\vec{\beta}}\left(\left\|U_{t}-\vec{\beta} U_{P}\right\|_{2}^{2}+\alpha\|\vec{\beta}\|_{1}\right),
$$

where $\alpha$ is the weight of the $L_{1}$ normalization and is set to be $10^{-4}$ in this paper. It is worth noting that the $L_{1}$ normalization is utilized in LASSO to further shrink the coefficient vector $\vec{\beta}_{P}$ to better distinguish the correct terms from error compensation terms. When the training epoch $>1000$, the term whose absolute value of coefficient is smaller than a threshold $\lambda$ will be dropped in each epoch. The value of the threshold $\lambda$ is fixed to be $10^{-4}$ in this paper. It is worth mentioning that, in previous work [25], the choice of threshold is crucial and must be changed frequently to discover different PDEs in different situations, especially when faced with high levels of noise, which means that a sophisticated procedure is needed to adjust the threshold. In comparison, in this paper, the threshold is fixed, which makes our proposed method more robust. Moreover, considering that LASSO will bring errors to the calculated coefficients, least square regression is employed for the rest of the epochs, which is written as

$$
\vec{\beta}_{P}=\left(U_{P}^{T} U_{P}\right)^{-1} U_{P}^{T} U_{t} .
$$

During the training process, the $\vec{\beta}_{P}$ is trained once per epoch. When the training epoch approaches the maximum epoch, the training process will be stopped, and the ultimate nonzero terms are the discovered terms, and $\vec{\beta}_{P}$ is the corresponding coefficient. It has been proven in previous work [25] that the correct physical constraint can assist to improve the derivative calculation of the neural network. In this paper, although the preliminary discovered potential terms by the generalized GA may be incorrect, they still contain underlying information and are close to the true physical process. Therefore, we construct an optimization cycle by the PINN, which is illustrated in Fig. 5. In the optimization cycle, the physical constraints (i.e., the discovered potential terms) make the neural network closer to the true physical process and thereby improve the accuracy of derivative calculation, while the improvement of derivative calculation will make the physical constraints more accurate. Overall, $\operatorname{PINN}(x, t ; \theta)$ utilizes the underlying physical information contained in the preliminary result of potential terms to further optimize the structure of the neural network to get closer to the actual physical processes, which improves the accuracy and stability of the discovered PDE. 


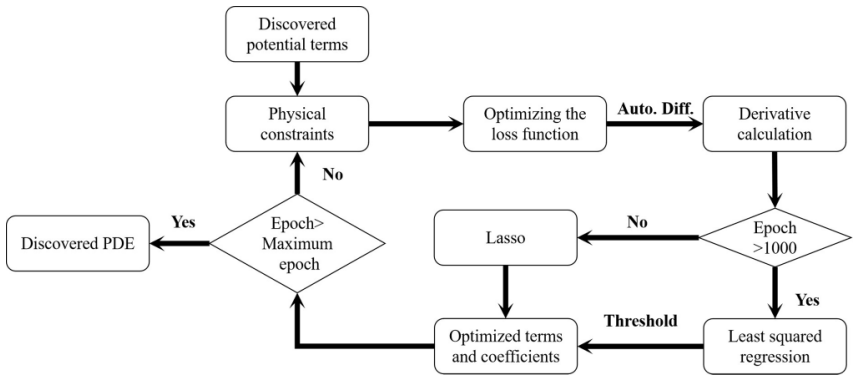

FIG. 5. The workflow for the optimization cycle in the physicsinformed neural network (PINN). Auto. Diff. refers to automatic differentiation.

\section{RESULTS}

In this section, the performance of our proposed R-DLGA in three typical complex situations is tested, including sparse data with high noise, high-order derivatives, and shock waves. The KdV equation, the KS equation, and the Burgers equation are taken as examples. These examples are employed to examine the robustness and accuracy of the R-DLGA when dealing with various difficult cases [28].

In this paper, two neural networks $\mathrm{NN}(x, t ; \theta)$ and $\operatorname{PINN}(x, t ; \theta)$ both have five layers: an input layer, an output layer, and three hidden layers, with 50 neurons in each hidden layer. The maximum training epoch for $\mathrm{NN}(x, t ; \theta)$ and $\operatorname{PINN}(x, t ; \theta)$ is 50000 and 20000 , respectively. It is worth noting that the selection of the maximum training epoch is determined by both experience and pretest and is not strict. All datasets are separated into training data and validate data to compute loss values and conduct early termination to prevent overfitting. The activation function is $\sin (x)$. The value of the threshold is $10^{-4}$. For the generalized GA, the population size of genomes is 400 , and the number of maximum generations is 100 . The rate of crossover is 0.8 , the rate of order mutation and basic gene mutation is 0.3 , the rate of delete-module mutation is 0.5 , and the rate of add-module mutation is 0.4 . The $l_{1} \_$norm in LASSO is $10^{-4}$. The hyperparameter $\varepsilon$ is
0.1 for the $\mathrm{KdV}$ equation, and 0.01 for the $\mathrm{KS}$ and Burgers equations.

\section{A. Discovery of KdV equation from sparse data with high noise}

The KdV equation is a PDE discovered by Korteweg and de Vries [29] to describe the motion of unidirectional shallow water, which is expressed as

$$
u_{t}=-u u_{x}-0.0025 u_{x x x} .
$$

The dataset is generated by numerical simulation with 512 spatial observation points in the domain $x \in[-1,1)$ and 201 temporal observation points in the domain $t \in[0,1]$. Therefore, the data volume is 102912 . For metadata, there are 400 spatial points in the domain $x \in[-0.8,0.8]$ and 300 temporal observation points in the domain $t \in[0.1,0.9]$, and thus, the total number of metadata is 120000 . To better demonstrate the stability and accuracy of the R-DLGA facing sparse and noisy data, 25000 (24.4\% of the total data volume), $10000(9.80 \%)$, 2500 (2.44\%), 1000 (1.22\%), 500 (0.44\%), and $100(0.09 \%)$ data are randomly selected to form new datasets. Here, $0 \%$ noise (clean data), $5 \%$ noise, $15 \%$ noise, $25 \%$ noise, and $50 \%$ noise are added to the dataset in the following form:

$$
u(x, t)=u(x, t) \cdot(1+\gamma \times e),
$$

where $\gamma$ denotes the noise level, and $e$ is a uniform random variable, taking values from -1 to 1 . The result is shown in Fig. 6(a). Here, the relative error is calculated in the following way:

$$
\text { error }=\left(\frac{\sum_{j=1}^{N_{t}} \sum_{i=1}^{N_{x}}\left|u\left(x_{i}, t_{j}\right)-u^{\prime}\left(x_{i}, t_{j}\right)\right|^{2}}{\sum_{j=1}^{N_{t}} \sum_{i=1}^{N_{x}}\left|u\left(x_{i}, t_{j}\right)\right|^{2}}\right)^{1 / 2} \times 100 \%,
$$

where $u\left(x_{i}, t_{j}\right)$ is the solution of the correct PDE, $u^{\prime}\left(x_{i}, t_{j}\right)$ is the solution of the discovered PDE, and $N_{x}$ and $N_{t}$ are the number of $x$ and $t$, respectively. Meanwhile, the discovered PDEs from these new noisy datasets by the DLGA alone are illustrated in Fig. 6(b) for comparison. The DLGA method has been compared with other PDE discovery methods on the $\mathrm{KdV}$ equation in our previous work [14] and has shown (a)

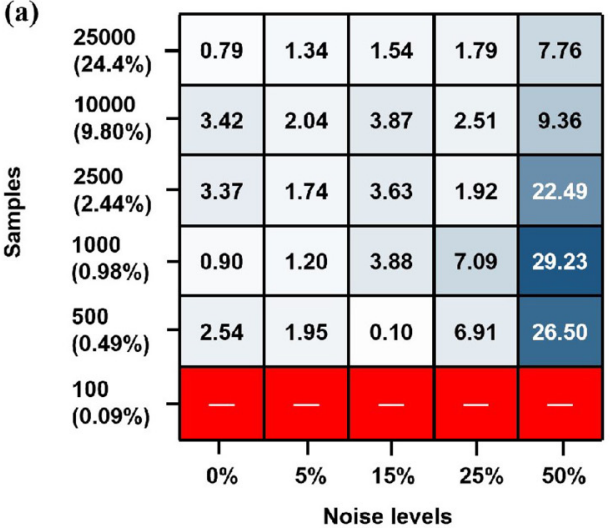

(b)

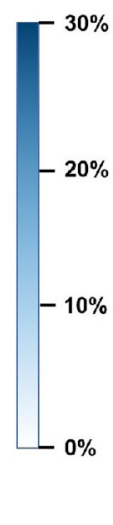

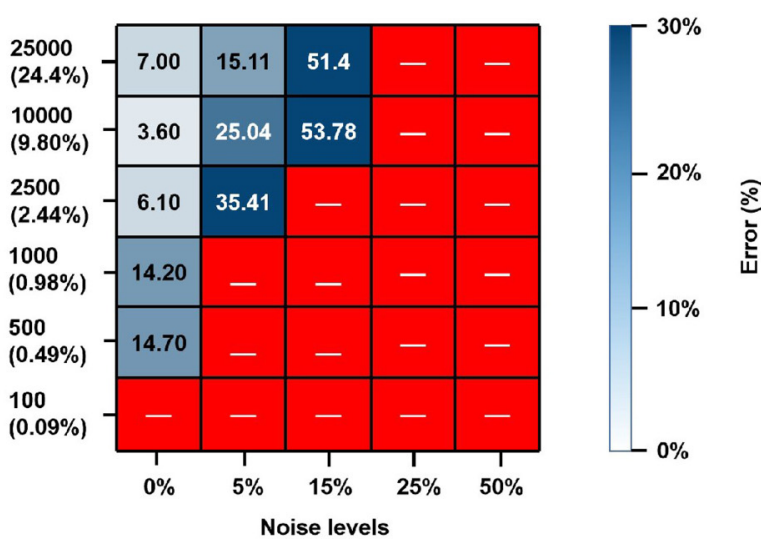

FIG. 6. The identified Korteweg-de Vries (KdV) equation with different volumes of data and different levels of noise through (a) the robust deep-learning genetic algorithm (R-DLGA) and (b) the DLGA alone. The red grid indicates that an incorrect partial differential equation (PDE) is discovered, while the blue grid indicates that a correct PDE is discovered. The value in the grid refers to the relative error. Here, the darker the blue color, the larger the relative error. 
TABLE I. The KS equation identified by the R-DLGA with different levels of noise added to the data.

\begin{tabular}{lcc}
\hline \hline & Correct PDE: $u_{t}=-u u_{x}-u_{x x}-u_{x x x x}$ & \\
\hline Noise level & Learned equation & Error \\
\hline Clean data & $u_{t}=-0.999 u u_{x}-1.000 u_{x x}-1.000 u_{x x x x}$ & $2.93 \%$ \\
1\% noise & $u_{t}=-0.995 u u_{x}-0.994 u_{x x}-0.994 u_{x x x x}$ & $3.16 \%$ \\
5\% noise & $u_{t}=-0.992 u u_{x}-0.992 u_{x x}-0.992 u_{x x x x}$ & $6.12 \%$ \\
$10 \%$ noise & $u_{t}=-0.325 u u_{x}-0.288 u_{x x}-0.195 u$ & - \\
\hline \hline
\end{tabular}

relatively high stability and robustness. Therefore, it is chosen as the benchmark to better illustrate the performance of the R-DLGA here. From the figure, it is observed that, although the DLGA alone can discover PDEs with relatively high levels of noise ( $15 \%$ noise), the error is large. In contrast, the RDLGA is surprisingly robust to small data volume and high noise. Indeed, the outcome remains stable and accurate even with 500 data $(0.49 \%)$ and $50 \%$ noise. Moreover, under the same conditions, the error of PDE discovered by the R-DLGA is much smaller, especially when the noise is high, and the amount of data is small. This demonstrates that the physical constraints in the PINN process provided by the preliminary result of potential terms can improve the performance of derivative calculation, which enables our proposed algorithm to be more robust to small data volume with large data noise.

\section{B. Discovery of KS equation with high-order derivatives}

In this part, the ability of the R-DLGA to discover PDEs with high-order derivatives is tested with the KS equation, the form of which is expressed as follows:

$$
u_{t}=-u u_{x}-u_{x x}-u_{x x x x} .
$$

The dataset is generated by numerical simulation with 512 spatial observation points in $x \in[-10,10)$ and 251 temporal observation points in $t \in[0,50]$. As a result, the total number of data is 128512 . For metadata, there are 400 spatial points in $x \in[-6,6]$ and 300 temporal observation points in $t \in[10,40]$, and thus, the total number of metadata is 120000 . The KS equation has a fourth-order derivative which is difficult to calculate accurately through existing derivative calculation methods, especially with noisy data. In this experiment, 60000 data are randomly selected to train the neural network, and $0 \%$ noise (clean data), $1 \%$ noise, $5 \%$ noise, and $10 \%$ noise are added to the data. The result is shown in Table I.

Until now, the best result is obtained by the integral form [30], which decreases the derivative order to the third order, which is easier to calculate and is robust to 5\% noise. However, although the PDE form can be discovered, the accuracy is still unsatisfactory, with $25 \%$ relative error for clean data and $95 \%$ relative error for $5 \%$ noise. In comparison, the error of the PDE discovered by the R-DLGA is much smaller, with $2.93 \%$ relative error for clean data and $6.12 \%$ relative error for 5\% noise. This indicates that the R-DLGA can calculate high-order derivatives accurately by adding the preliminary result of potential terms to the loss function of the PINN as physical constraints. Additional details about the comparison with existing PDE discovery methods for discovering the KS equation are provided in Appendix A.

\section{Discovery of Burgers equation with shock waves}

The Burgers equation has been identified many times for proof of concept in previous works [2,10], and its form is written as

$$
u_{t}=-u u_{x}+a u_{x x},
$$

where $a$ is the coefficient of the viscous term. In previous investigations [2,10], $a$ is set to be 0.1 , and the solution is smooth without a shock wave. In this paper, a more challenging situation is considered in which $a$ is set to be $0.01 / \pi$, which means that the viscous term is so small that a shock wave will emerge. The dataset is the same as that in Both et al. [25]. For the dataset, there are 256 spatial observation points in $x \in[-1,1]$ and 100 temporal observation points in $t \in$ $[0,1)$. Consequently, the total number of data is 25600 . For metadata, there are 400 spatial points in $x \in[-0.8,0.8]$ and 300 temporal observation points in $t \in[0.1,0.9]$, and thus, the total number of metadata is 120000 . Here, 5000 data $(19.53 \%$ of the total data volume) are randomly selected to train the neural network, and $0 \%$ noise (clean data) and 25\% noise are added to the data. The results are displayed in Figs. 7(a) and 7(b), respectively. From the figure, the discovered PDE is close to the true PDE, and the shock wave is recovered with high accuracy, which means that the R-DLGA can handle the PDE with shock waves even though the derivative calculation is difficult at the location of the shock wave. In addition, it is found that the performance of the R-DLGA when identifying the Burgers equation with a shock wave is more robust to data noise compared with previous work [24] that is robust to $10 \%$ noise. Additional details about the comparison with existing PDE discovery methods for discovering the Burgers equation with a shock wave are provided in Appendix A.

\section{Effect of the generalized GA}

In this paper, a unique GA, called the generalized GA, is proposed to obtain a preliminary result of potential terms, in which terms are expressed in the compound form comprised of an inner term and a derivative order. With the generalized $\mathrm{GA}$, an interesting phenomenon is discovered. The KdV equation with 2500 data training the neural network is taken as an example again, and different levels of noise are added to the data. The results of the generalized GA, including discovered potential term and corresponding coefficients, are presented in Table II. For comparison, the ultimate discovered PDE by the R-DLGA is also provided in Table II. From the table, the identified potential PDE terms are correct when the noise level is low, while redundant potential terms are found when the noise level is high. However, it is surprising to find that several high-order terms with tiny coefficients [e.g., $u_{x x x x x}$ and $\left(u^{2}\right)_{x x x}$ ] occur in the potential terms, while the correct terms are also in the potential terms with their coefficients being relatively accurate. This means that these high-order terms function as error compensation terms which may compensate the error resultant from derivative calculation. 
(a)

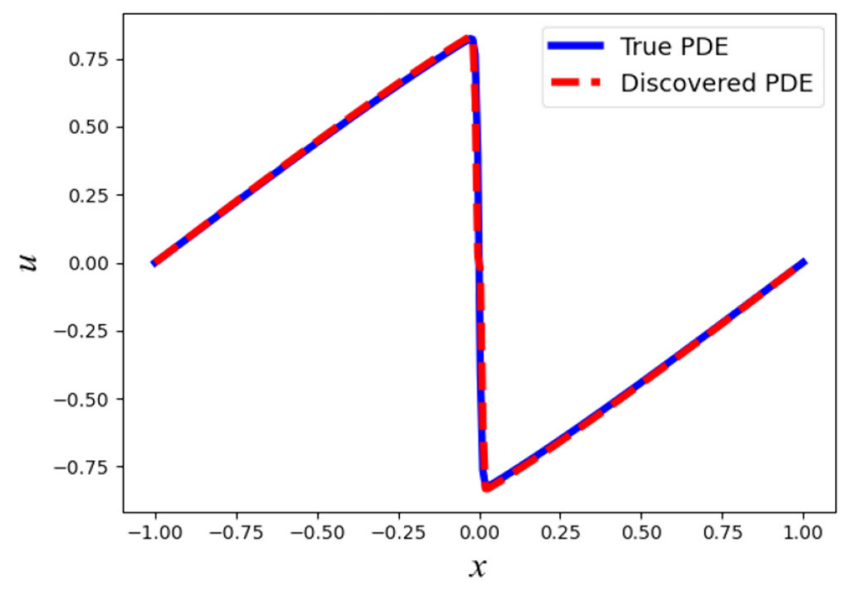

$u_{t}=-0.993 u u_{x}+0.0034 u_{x x}$ (b)

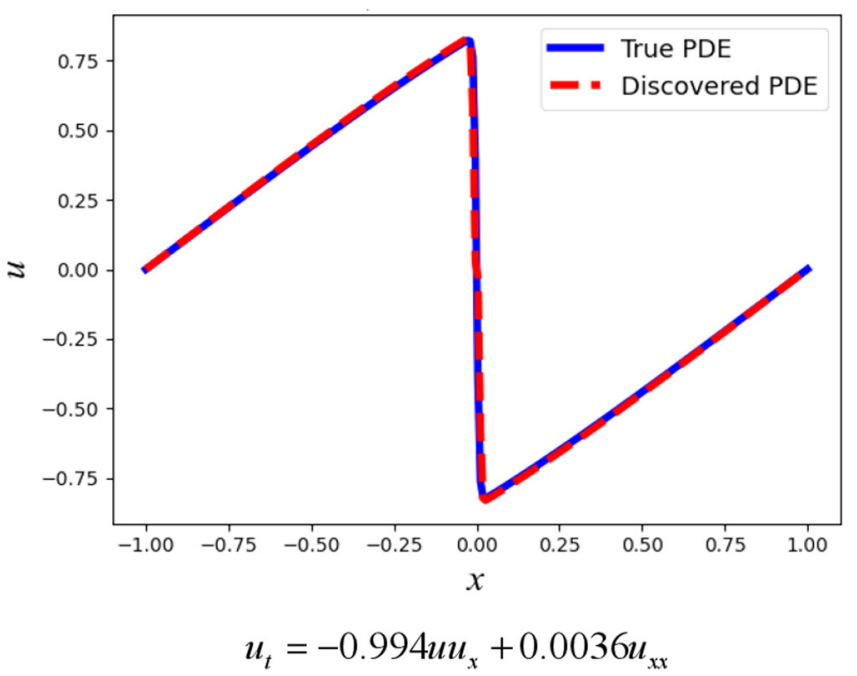

FIG. 7. The solution of the identified partial differential equation (PDE) and the true PDE for the Burgers equation with a shock wave when $t=0.8$ with (a) clean data and (b) $25 \%$ noise.

The emergence of error compensation terms is an interesting phenomenon that is rarely revealed in previous works. To further investigate the effect of the error compensation terms discovered by the generalized GA, different PDE discovery methods, including STRidge, the common GA, the generalized GA, and the R-DLGA, are adopted to discover the $\mathrm{KdV}$ equation with 2500 data and $25 \%$ noise. The results are presented in Table III. It can be found that only the R-DLGA discovered the correct PDE, and the coefficients are accurate, while the others fail. Among them, STRidge is completely incorrect, and although the true terms are also contained in the PDE discovered by the common GA, their coefficients are far from the correct coefficients. In contrast, the coefficients of the correct term discovered by the generalized GA are relatively accurate. From the comparison, it is revealed that the discovered error compensation terms are not arbitrary since, although the common GA also identifies correct terms and redundant terms, the coefficients of true terms are inaccurate. Additional experiments are conducted in Appendix A, and the results show that error compensation terms also emerge when identifying the Burgers equation with a shock wave, and the identified true terms are relatively accurate as well. Therefore, it is supposed that the error compensation terms can compensate the error brought by these complex situations (e.g., high noise, shock wave) and keep the coefficients of correct terms relatively accurate. It will guarantee the stability of the identification of the correct terms in the potential terms, which is significant for the PINN step. This interesting phenomenon can be discovered because the generalized GA adds the derivative order into the gene module and attempts to find a compound form which is more concise and flexible, so that the genome can automatically increase the derivative order and generate complex terms via mutation to identify a better solution. In contrast, previous methods usually consider up to fourth-order derivatives at most, and only a simple form can be generated, and thus, it is difficult to find suitable error compensation terms.

\section{E. The effect of PINN steps}

In this part, the effect of PINN steps is investigated. The $\mathrm{KdV}$ equation with $50 \%$ noise utilizing 500 data and 2500 data training the neural network and the Burgers equation with 5000 data with $25 \%$ noise and $0 \%$ noise are examined again. True data, noisy data, and the outcome of $\mathrm{NN}(x, t ; \theta)$ and $\operatorname{PINN}(x, t ; \theta)$ are plotted in Fig. 8. From the figure, numerous findings are evident. Firstly, with a high level of noise, the noisy observation data have a large derivation compared with the true data, and the neural network can learn the underlying physical dynamics from the sparse data with high noise. This

TABLE II. Potential terms and corresponding coefficients discovered by the generalized GA and ultimately learned PDE by the R-DLGA with different levels of noise added to the data for discovering the $\mathrm{KdV}$ equation.

\begin{tabular}{llr}
\hline \hline \multicolumn{1}{c}{ Correct PDE: $u_{t}=-0.0025 u_{x x x}-u u_{x}$} \\
\hline Noise level & \multicolumn{1}{c}{ Learned equation by generalized GA } & Learned equation by R-DLGA \\
\hline Clean data & $u_{t}=-0.00247 u_{x x x}-0.494\left(u^{2}\right)_{x}$ & $u_{t}=-0.00249 u_{x x x}-0.993 u u_{x}$ \\
$5 \%$ noise & $u_{t}=0.00231 u_{x x x}-0.466\left(u^{2}\right)_{x}$ & $u_{t}=-0.00250 u_{x x x}-1.002 u u_{x}$ \\
$15 \%$ noise & $u_{t}=-0.00230 u_{x x x}-0.447\left(u^{2}\right)_{x}-2.11 \times 10^{-7}\left(u_{x x}\right)_{x x x}$ & $u_{t}=-0.00249 u_{x x x}-0.993 u u_{x}$ \\
$25 \%$ noise & $u_{t}=-0.00247 u_{x x x}-0.994 u u_{x}-8.67 \times 10^{-7}\left(u_{x x}\right)_{x x x}-1.68 \times 10^{-4}\left(u^{2}\right)_{x x x}$ & $u_{t}=-0.00250 u_{x x x}-0.997 u u_{x}$ \\
$50 \%$ noise & $u_{t}=-0.00225 u_{x x x}-0.895 u u_{x}-9.92 \times 10^{-7}\left(u_{x x}\right)_{x x x}-1.86 \times 10^{-4}\left(u^{2}\right)_{x x x}$ & $u_{t}=-0.00244 u_{x x x}-0.954 u u_{x}$ \\
\hline \hline
\end{tabular}


TABLE III. PDE discovered by different methods for the KdV equation from sparse data with high noise.

\begin{tabular}{ll}
\hline \hline & Correct PDE: $u_{t}=-0.0025 u_{x x x}-u u_{x}$ \\
\hline Learned equation by STRidge & $u_{t}=-0.807 u u_{x}$ \\
Learned equation by common GA & $u_{t}=-0.00129 u_{x x x}-0.447 u u_{x}-3.01 \times 10^{-4} u_{x}^{3}$ \\
Learned equation by generalized GA & $u_{t}=-0.00247 u_{x x x}-0.994 u u_{x}-8.67 \times 10^{-7}\left(u_{x x}\right)_{x x x}-1.68 \times 10^{-4}\left(u^{2}\right)_{x x x}$ \\
Learned equation by R-DLGA & $u_{t}=-0.00250 u_{x x x}-0.997 u u_{x}$ \\
\hline \hline
\end{tabular}

indicates that the DLGA steps can identify the potential terms with high confidence. However, the figure also shows that the outcome of $\mathrm{NN}(x, t ; \theta)$ still has some deficiencies. For example, certain deviations will occur when faced with sparse data with high levels of noise [see Fig. 8(a)], and oscillations will emerge near the shock wave [see Fig. 8(c)]. This limitation will bring a certain error to derivative calculation, which finally leads to the failure of PDE discovery when faced with complex situations, and therefore, the PINN steps are utilized here. The figure reveals that, after adding the potential terms discovered by the DLGA steps as physical constraints, the trained PINN is closer to the true data even with high noise and shock waves, which maintains the accuracy of derivative calculation and improves the performance of PDE discovery. Finally, when the noise level is low or the amount of data is relatively large [see Figs. 8(b) and $8(\mathrm{~d})$ ], $\mathrm{NN}(x, t ; \theta)$ performs well with a little error, while the outcome of PINN has filtered these errors and is perfectly compliant with the true data. (a)

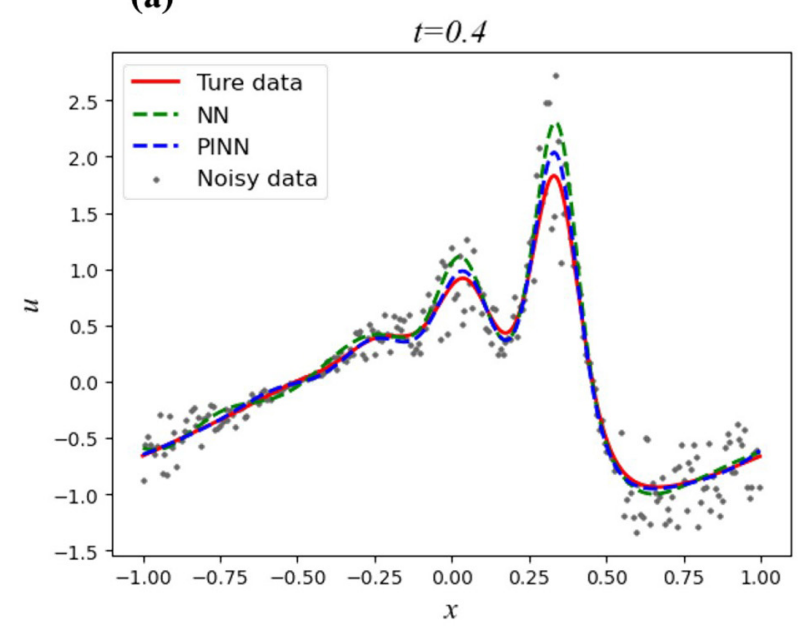

(c)

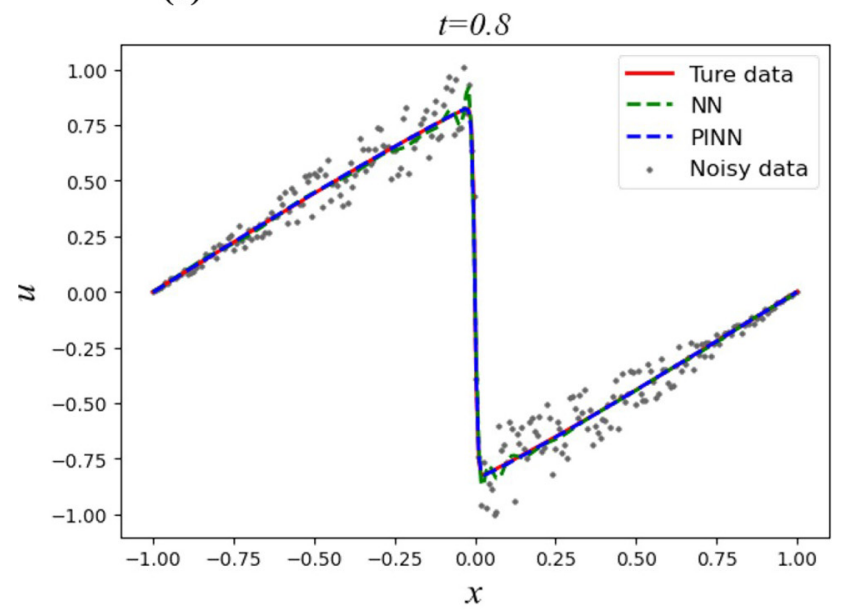

(b)

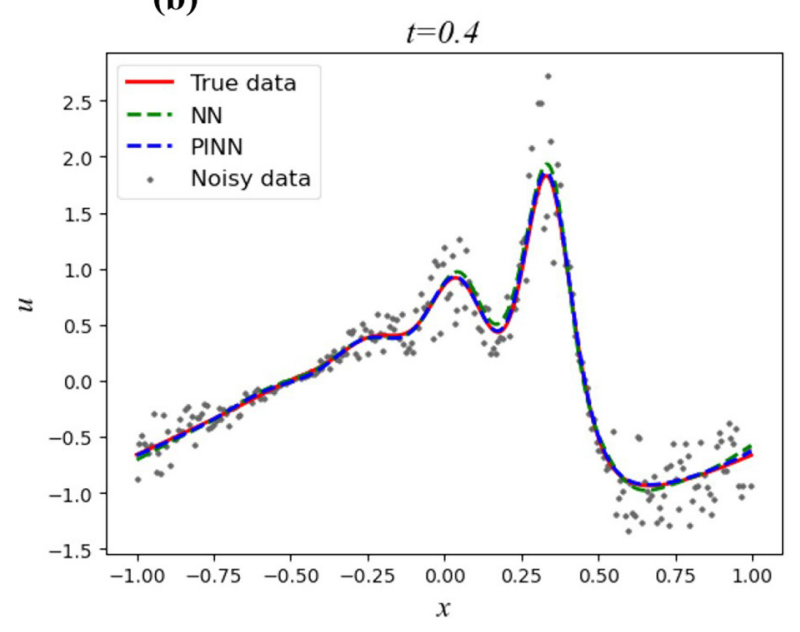

(d)

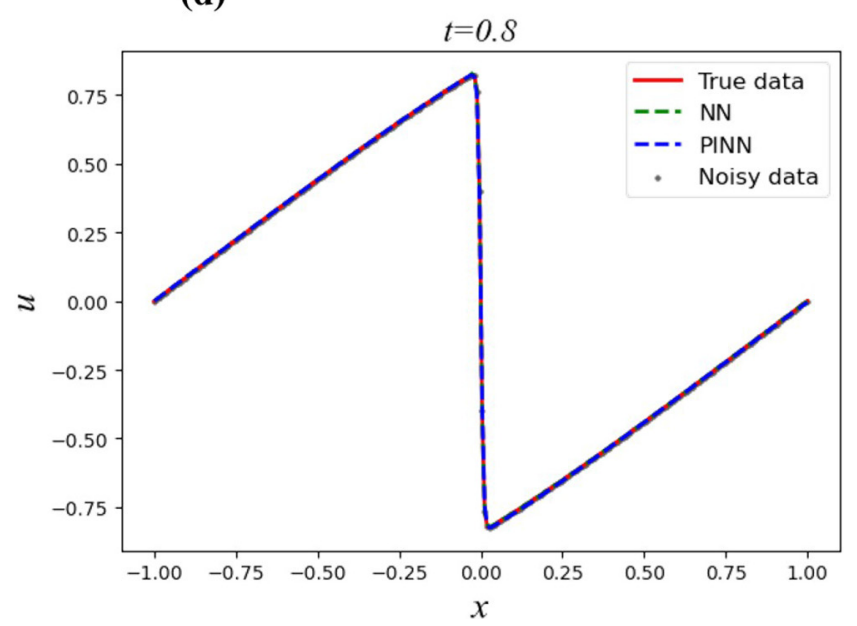

FIG. 8. True data, noisy data, and the outcome of $\mathrm{NN}(x, t ; \theta)$ and the physics-informed neural network PINN $(x, t ; \theta)$ for the Korteweg-de Vries (KdV) equation at $t=0.4$ with (a) 500 data and (b) 2500 data training the neural network, and the Burgers equation with a shock wave $t=0.8$ with (c) $25 \%$ noise and (d) clean data. Here, noisy data are plotted on each point of $x$ to better illustrate the data noise, and the utilized noisy data are sparser. 
TABLE IV. PDE discovered by different methods for the KS equation from sparse data with clean data and 5\% noise.

\begin{tabular}{lcc}
\hline \hline & \multicolumn{1}{c}{ Correct PDE: $u_{t}=-u u_{x}-u_{x x}-u_{x x x x}$} & \\
\hline Methods & Clean data & $5 \%$ noise \\
\hline STRidge & $u_{t}=-0.552 u u_{x}-0.442 u_{x x}-0.478 u_{x x x x}$ & $u_{t}=-0.488 u u_{x}-0.329 u_{x x}-0.374 u_{x x x x}$ \\
Common GA & $u_{t}=-0.555 u u_{x}+0.190 u u_{x}^{2}-0.665 u_{x} u_{x x}$ & $u_{t}=-0.487 u u_{x}-0.207 u-0.289 u_{x x x x}$ \\
Generalized GA & $u_{t}=-0.554 u u_{x}-0.443 u_{x x}-0.480 u_{x x x x}$ & $u_{t}=-0.256\left(u^{2}\right)_{x}-0.372 u_{x x}-0.415 u_{x x x x}$ \\
R-DLGA & $u_{t}=-0.999 u u_{x}-1.000 u_{x x}-1.000 u_{x x x x}$ & $u_{t}=-0.992 u u_{x}-0.992 u_{x x}-0.992 u_{x x x x}$ \\
\hline \hline
\end{tabular}

\section{DISCUSSION AND CONCLUSIONS}

In this paper, a framework called the R-DLGA is proposed to handle complex situations, including sparse data with high noise, high-order derivatives, and shock waves, which is difficult to deal with by existing methods. In the framework, a neural network is employed to learn a substitute model from the sparse and noisy data, and a GA called the generalized GA is proposed to give a preliminary result of potential terms that is then added into the loss function of the PINN as physical constraints. The PINN is employed to further optimize the structure of the neural network to get closer to the actual physical processes and improve the accuracy of derivative calculation. A threshold is utilized to drop error compensation terms identified in the potential terms during the optimization process, and the reserved terms and their corresponding coefficients are the ultimate discovered PDE. The KdV equation, the KS equation, and the Burgers equation with a shock wave are employed to test our proposed algorithm. The results demonstrate that the R-DLGA is stable and accurate in complex situations, including sparse data with high noise, high-order derivatives, and shock waves.

In the field of PDE discovery, the main concern is derivative calculation, which directly determines whether the correct PDE can be identified and the accuracy of the discovered PDE. However, faced with the complex situations mentioned above, derivatives are difficult to be calculated accurately enough to discover the correct PDE, even if automatic differentiation is employed. Our proposed algorithm, however, finds another approach to improve the accuracy and stability of derivative calculation, which combines the DLGA and the PINN. Numerical experiments have shown that the neural network $\mathrm{NN}(x, t ; \theta)$ can learn the underlying dynamics to a certain extent but is not sufficiently precise to directly discover the correct PDE in complex situations. Therefore, our proposed generalized GA, which involves an inner term and a derivative order in the gene module, is utilized to discover potential terms. Different from the traditional GA, the generalized GA aims to discover a compound form of PDE terms which is more concise and flexible. Therefore, the same term will have several different equivalent forms, which can increase the stability of the algorithm, and additional details about the influence of the equivalent forms are provided in Appendix B. Meanwhile, order mutation enables the generalized GA to automatically add high-order derivatives if low-order derivatives are insufficient. It is worth noting that the values of PDE terms in compound form are calculated via the finite difference method, which means that high-order derivatives do not need to be previously defined and calculated since they can be calculated automatically based on several basic genes. Consequently, our proposed algorithm can discover PDEs in a wide range of variation libraries and solve the problem of an incomplete candidate library. Results have also demonstrated that the generalized GA can identify the correct PDE terms with relatively accurate coefficients. Furthermore, several high-order error compensation terms with tiny coefficients are found, which means that the generalized GA is stable to contain the correct terms in the potential terms, and it will pave the way for the PINN steps. It is worth mentioning that the error compensation terms were rarely discovered and noted previously. This is because sparse regression techniques (e.g., LASSO, STRidge) employed in previous works [1-4] attempt to obtain a parsimonious model by employing the $l_{1}$ or $l_{2}$ penalty to shrink and drop tiny coefficients. However, the coefficients of error compensation terms are usually tiny and will be dropped during the sparse regression process.

In this paper, numerical experiments are carried out to examine the effect of the PINN steps, and the results prove that the physical constraints provided by potential terms can significantly increase the accuracy of derivatives calculation since it is closer to the underlying physical process even with shock waves and high noise. Different from the DeepMod method proposed by Both et al. [25], in which a predetermined complete candidate library with numerous terms is defined

TABLE V. PDE discovered by different methods for the Burgers equation with a shock wave from sparse data with clean data and $25 \%$ noise.

\begin{tabular}{|c|c|c|}
\hline \multicolumn{3}{|c|}{ Correct PDE: $u_{t}=-u u_{x}+\frac{0.01}{\pi} u_{x x}$} \\
\hline Methods & Clean data & $25 \%$ noise \\
\hline STRidge & $u_{t}=-0.077 u u_{x}$ & $u_{t}=-0.058 u u_{x}$ \\
\hline Common GA & $u_{t}=-0.784 u u_{x}+0.00248 u_{x x}$ & $u_{t}=-0.308 u u_{x}-4.2 \times 10^{-5} u_{x} u_{x x}$ \\
\hline Generalized GA & $\begin{aligned} u_{t}= & -0.990 u u_{x}+0.0031\left(u_{x}\right)_{x}+3.94 \times 10^{-8}\left(u_{x}\right)_{x x x} \\
& -8.0 \times 10^{-6}\left(u^{2}\right)_{x x x}\end{aligned}$ & $\begin{aligned} u_{t}= & -0.777 u u_{x}+0.0018\left(u_{x}\right)_{x} \\
& -6.23 \times 10^{-4}\left(u u_{x}\right)_{x x}\end{aligned}$ \\
\hline R-DLGA & $u_{t}=-0.993 u u_{x}+0.0034 u_{x x}$ & $u_{t}=-0.994 u u_{x}+0.0036 u_{x x}$ \\
\hline
\end{tabular}


for physical constraints, our proposed algorithm employed the generalized GA to identify a few potential terms as physical constraints. This makes our optimization cycle more efficient, and the selection of the threshold $\lambda$ in the PINN is not crucial because it is easier to maintain sparsity since terms in the physical constraints are parsimonious. In addition, different PDE discovery methods are adopted to discover the $\mathrm{KdV}$ equation from sparse data with high noise. The result shows that existing methods still possess certain defects since their performances are unsatisfactory, while the R-DLGA succeeds in discovering the correct PDEs with high accuracy.

Overall, our proposed R-DLGA algorithm is robust and accurate in various complex conditions, such as sparse data with noise, high-order derivatives, and shock waves. Moreover, it can discover PDEs from a large variation library and solve the problem of an incomplete candidate library. Despite these advantages, our proposed algorithm still possesses certain limitations and challenges. Firstly, although our proposed method can discover PDEs stably and accurately, the current research is still in the stage of proof of concept. Therefore, applications in practical problems require further investigation. Secondly, only one-dimensional PDEs are investigated. Since discovery of PDEs with higher dimensions in complex situations is more challenging, further improvement of the R-DLGA to suit high-dimensional PDEs is needed. Finally, if the underlying physical process has high complexity (e.g., multiscale problems), the PINN employed in this paper may be hard to train sufficiently well to obtain ultimate outcomes. Further works regarding these issues are necessary.

\section{ACKNOWLEDGMENTS}

This paper is partially funded by the Shenzhen Key Laboratory of Natural Gas Hydrates (Grant No. ZDSYS20200421111201738) and the SUSTech-Qingdao New Energy Technology Research Institute.

\section{APPENDIX A: COMPARISON WITH EXISTING METHODS}

In Sec. III D, the performance of our proposed generalized GA and the R-DLGA on the discovery of the KdV equation is compared with existing methods to demonstrate the effect of the generalized GA. In this part, the KS equation and the Burgers equation with a shock wave are also investigated. For the KS equation, 60000 data are randomly selected to train the neural network with no noise and 5\% noise. For the Burgers equation with a shock wave, 5000 data with clean data and $25 \%$ noise are randomly selected to train the neural network. Different PDE discovery methods, including STRidge, the common GA, the generalized GA, and the R-DLGA, are employed to discover the underlying PDE from metadata
TABLE VI. The fitness of four equivalent forms of the KdV equation and corresponding coefficients calculated in the GA.

\begin{tabular}{llc}
\hline \hline Genome & \multicolumn{1}{c}{ Equation } & Fitness \\
\hline$\{[(0): 3],[(0,1): 0]\}$ & $u_{t}=-0.002424 u_{x x x}-0.9702 u u_{x}$ & 0.4079 \\
$\{[(0): 3],[(0,0): 1]\}$ & $u_{t}=-0.002412(u)_{x x x}-0.4854\left(u^{2}\right)_{x}$ & 0.4085 \\
$\{[(3): 0],[(0,1): 0]\}$ & $u_{t}=-0.002415 u_{x x x}-0.9699 u u_{x}$ & 0.4092 \\
$\{[(3): 0],[(0,0): 1]\}$ & $u_{t}=-0.002421 u_{x x x}-0.4856\left(u^{2}\right)_{x}$ & 0.4068 \\
\hline \hline
\end{tabular}

generated by the neural network. The results are displayed in Tables IV and V.

From Table IV, although STRidge can also discover true terms, the accuracy is lower than the generalized GA. However, due to the existence of the fourth-order derivative, the accuracy of the generalized GA is still unsatisfied when data are noisy. Therefore, the PINN process is needed to obtain a more accurate and stable result. From Table V, the error compensation term is discovered by the generalized GA, which guarantees that the true terms are discovered, while STRidge and the common GA both fail to discover true terms when the noise level is high. Meanwhile, experiments also show that the effect of the generalized GA cannot be ignored even with clean data because other existing methods are not sufficiently stable to identify true terms when faced with these complex situations.

\section{APPENDIX B: EQUIVALENT FORM OF TERMS IN THE GENERALIZED GA}

In the proposed generalized GA, terms are expressed in compound form, which comprises the inner term and the derivative order. Therefore, the same term will have several different equivalent forms. For example, the term $u u_{x}$ may be expressed as $[(0,0): 1]$ that refers to $\left(u^{2}\right)_{x}$, or $[(0,1): 0]$ that refers to $u u_{x}$, both of which are equivalent. In this part, the influence of the equivalent form of terms in the GA is investigated, and the $\mathrm{KdV}$ equation is taken as an example. Here, 25000 data are randomly selected to generate the new dataset, and 5\% noise is added to the dataset. The correct PDE has several equivalent forms, and four of them are selected as examples. The fitness of different equivalent forms of the correct PDE and corresponding coefficients calculated in the GA are presented in Table VI. From the table, the fitness of the equivalent forms and corresponding coefficients are slightly different. The common GA can only discover the form in the first row $\left(u_{x x x}\right.$ and $\left.u u_{x}\right)$; however, our proposed generalized GA is able to discover an equivalent form with smaller (better) fitness, which is more accurate and stable.
[1] H. Schaeffer, Learning partial differential equations via data discovery and sparse optimization, Proc. R. Soc. A 473, 20160446 (2017).
[2] S. H. Rudy, S. L. Brunton, J. L. Proctor, and J. N. Kutz, Datadriven discovery of partial differential equations, Sci. Adv. 3, e1602614 (2017). 
[3] S. Zhang and G. Lin, Robust data-driven discovery of governing physical laws with error bars, Proc. R. Soc. A 474, 20180305 (2018).

[4] S. L. Brunton, J. L. Proctor, J. N. Kutz, and W. Bialek, Discovering governing equations from data by sparse identification of nonlinear dynamical systems, Proc. Natl. Acad. Sci. USA 113, 3932 (2016).

[5] E. Kaiser, J. N. Kutz, and S. L. Brunton, Sparse identification of nonlinear dynamics for model predictive control in the low-data limit, Proc. R. Soc. A 474, 20180335 (2018).

[6] L. Boninsegna, F. Nüske, and C. Clementi, Sparse learning of stochastic dynamical equations, J. Chem. Phys. 148, 241723 (2018).

[7] M. Quade, M. Abel, J. Nathan Kutz, and S. L. Brunton, Sparse identification of nonlinear dynamics for rapid model recovery, Chaos 28, 063116 (2018).

[8] K. Champion, B. Lusch, J. Nathan Kutz, and S. L. Brunton, Data-driven discovery of coordinates and governing equations, Proc. Natl. Acad. Sci. USA 116, 22445 (2019).

[9] M. Raissi, P. Perdikaris, and G. E. Karniadakis, Physicsinformed neural networks: a deep learning framework for solving forward and inverse problems involving nonlinear partial differential equations, J. Comput. Phys. 378, 686 (2019).

[10] M. Raissi, Deep hidden physics models: deep learning of nonlinear partial differential equations, J. Mach. Learn. Res. 19, 1 (2018).

[11] Z. Long, Y. Lu, and B. Dong, PDE-Net 2.0: learning PDEs from data with a numeric-symbolic hybrid deep network, J. Comput. Phys. 399, 108925 (2019).

[12] H. Xu, H. Chang, and D. Zhang, DL-PDE: deep-learning based data-driven discovery of partial differential equations from discrete and noisy data, Commun. Comput. Phys. 29, 698 (2021).

[13] J. Berg and K. Nyström, Data-driven discovery of PDEs in complex datasets, J. Comput. Phys. 384, 239 (2019).

[14] H. Xu, H. Chang, and D. Zhang, DLGA-PDE: discovery of PDEs with incomplete candidate library via combination of deep learning and genetic algorithm, J. Comput. Phys. 418, 109584 (2020).

[15] H. Chang and D. Zhang, Machine learning subsurface flow equations from data, Comput. Geosci. 23, 895 (2019).

[16] M. Maslyaev, A. Hvatov, and A. Kalyuzhnaya, , in Computational Science - ICCS 2019, edited by J. M. F. Rodrigues, P. J. S. Cardoso, J. Monteiro, R. Lam, V. V Krzhizhanovskaya, M. H.
Lees, J. J. Dongarra, and P. M. A. Sloot (Springer International Publishing, Cham, 2019), pp. 635641.

[17] S. Atkinson, W. Subber, L. Wang, G. Khan, P. Hawi, and R. Ghanem, Data-driven discovery of free-form governing differential equations, arXiv:1910.05117.

[18] H. Xu, D. Zhang, and J. Zeng, Deep-learning of parametric partial differential equations from sparse and noisy data, Phys. Fluids 33, 037132 (2021).

[19] S. Rudy, A. Alla, S. L. Brunton, and J. N. Kutz, Data-driven identification of parametric partial differential equations, SIAM J. Appl. Dyn. Syst. 18, 643 (2019).

[20] J. Bakarji and D. M. Tartakovsky, Data-driven discovery of coarse-grained equations, J. Comput. Phys. 434, 110219 (2021).

[21] Z. Zhang and Y. Liu, Robust data-driven discovery of partial differential equations under uncertainties, arXiv:2102.06504.

[22] K. Wu and D. Xiu, Data-driven deep learning of partial differential equations in modal space, J. Comput. Phys. 408, 109307 (2020).

[23] P. A. K. Reinbold, D. R. Gurevich, and R. O. Grigoriev, Using noisy or incomplete data to discover models of spatiotemporal dynamics, Phys. Rev. E 101, 010203(R) (2020).

[24] D. A. Messenger and D. M. Bortz, Weak SINDy for partial differential equations, J. Comput. Phys. 443, 110525 (2021).

[25] G. J. Both, S. Choudhury, P. Sens, and R. Kusters, DeepMoD: deep learning for model discovery in noisy data, J. Comput. Phys. 428, 109985 (2021).

[26] N. Wang, D. Zhang, H. Chang, and H. Li, Deep learning of subsurface flow via theory-guided neural network, J. Hydrol. 584, 124700 (2020).

[27] M. Rong, D. Zhang, and N. Wang, A Lagrangian dual-based theory-guided deep neural network, arXiv:2008.10159.

[28] The datasets utilized in this paper are provided as an open resource at https://gitee.com/xh251314/R_DLGA/ tree/master/Datasets. The codes for R-DLGA and discovery of PDEs investigated in this paper are also provided as an open resource at https://gitee.com/xh251314/R_DLGA/ tree/master/R_DLGA_Code.

[29] D. J. Korteweg and G. de Vries, On the change of form of long waves advancing in a rectangular canal, and on a new type of long stationary waves, Philos. Mag. 39, 422 (1895).

[30] H. Xu, D. Zhang, and N. Wang, Deep-learning based discovery of partial differential equations in integral form from sparse and noisy data, J. Comput. Phys. 445, 110592 (2021). 